\title{
High Grade Ductal Breast Carcinoma In Situ
}

National Cancer Institute

\section{Source}

National Cancer Institute. High Grade Ductal Breast Carcinoma In Situ. NCI Thesaurus. Code C7949.

A ductal carcinoma in situ characterized by the presence of neoplastic cells with severe dysplasia and the formation of micropapillary, cribriform, or solid patterns. The nuclei show marked pleomorphism and have prominent nucleoli. Mitotic activity is usually present. There is comedo-type of necrosis present in the ducts. The necrotic debris is surrounded by pleomorphic malignant cells. 